\title{
Allele-specific $\mathrm{N}$-glycosylation delays human surfactant protein B secretion in vitro and associates with decreased protein levels in vivo
}

\author{
Saija Taponen', Johanna M. Huusko', Ulla E. Petäjä-Repo'2, Reija Paananene, ${ }^{1,3}$ Susan H. Guttentag ${ }^{4}$, Mikko Hallman ${ }^{1,5}$ \\ and Ritva Haataja ${ }^{1,6}$
}

BACKGROUND: Surfactant protein B (SP-B) is essential for normal lung function, and decreased concentrations of SP-B have a deleterious effect on pulmonary outcome. SP-B levels may correlate with variations in the encoding gene (SFTPB). SFTPB single-nucleotide polymorphism lle131Thr affects proSP-B N-glycosylation in humans and the glycosylated Thr variant associates with pulmonary diseases.

METHODS: We analyzed SP-B levels in amniotic fluid samples for associations with SFTPB polymorphisms and generated cell lines expressing either proSP-B/131lle or proSP-B/131Thr for examining the effect of glycosylation on proSP-B secretion kinetics. To determine any transcription preference between lle131Thr allelic variants, we used heterozygous human lungs for allelic expression imbalance assays.

RESULTS: Protein levels correlated with lle131Thr genotype and the lowest SP-B levels were observed in Thr/Thr homozygotes. Our results suggest that lle131Thr variation-dependent $\mathrm{N}$-glycosylation associates with decreased levels of SP-B, which is secreted from fetal lung to amniotic fluid. Glycosylated proSP-B/131Thr was secreted from transfected cells at a lower rate than nonglycosylated proSP-B/131Ile. Expression levels of the mRNA variants were equal. Secretion of the glycosylated variant was thus delayed in vitro by a posttranscriptional mechanism

CONCLUSION: These data support the hypothesis that proSPB glycosylation due to lle131Thr variation may have a causal role in genetic susceptibility to acute respiratory distress.

$\mathbf{P}$ ulmonary surfactant, a complex mixture of phospholipids, neutral lipids, and proteins, is produced, assembled, and secreted by type 2 alveolar epithelial cells in the alveolar epithelial lining $(1,2)$. Its main function is to lower surface tension by forming a surface-active film, and absence or dysfunction of this film results in alveolar collapse during expiration. This is seen in premature infants with respiratory distress syndrome (RDS), which is caused mainly by a deficiency of pulmonary surfactant at the time of birth $(2,3)$. In addition, pulmonary surfactant is a critical component of the innate immune defense system (3).

Surfactant protein B (SP-B), a highly hydrophobic minor component of pulmonary surfactant, is crucial for normal lung function $(2,4-6)$. In addition to its surface tension-lowering properties and essential functions related to surfactant metabolism (7), SP-B has roles in host defense $(8,9)$. Mature SP-B can be detected in developing human lungs at $24 \mathrm{wk}$ of gestation, although only at $3 \%$ of adult levels. SP-B in amniotic fluid originates from the fetal lung (10).

Bioactive SP-B is a homodimer $\sim 18 \mathrm{kDa}$ in size. After signal peptide cleavage, and on its route from the endoplasmic reticulum (ER) to intracellular lamellar bodies, processing of $42-\mathrm{kDa}$ proSP-B to the mature $8-\mathrm{kDa}$ SP-B monomer involves several cleavage steps. The initial $\mathrm{NH}_{2}$-terminal cleavage, which results in a $25-\mathrm{kDa}$ intermediate, is independent of cell type, whereas the more distal processing stages are specific to type 2 cells and are performed by cell type-specific proteases $(6,11-13)$. The active peptide is stored in lamellar bodies and secreted with phospholipids to the airway lumen (14).

$\mathrm{N}$-glycosylation is an important co- and posttranslational modification that performs diverse cellular functions. Human proSP-B contains two sites for N-linked glycosylation: in the $\mathrm{NH}_{2}$ terminus at Asn129 and in the $\mathrm{COOH}$ terminus at Asn311 (6). The fragments of proprotein containing these sites are cleaved before the formation of mature SP-B polypeptide. Glycosylation at Asn129 is specific to humans and is dependent on the nonsynonymous single-nucleotide polymorphism Ile131Thr (rs1130866) in the SP-B-encoding gene SFTPB $(15,16)$.

The SFTPB 131Thr variant polypeptide is glycosylated, and the Thr allele is associated with several pulmonary diseases such as neonatal RDS, acute RDS in adults, and idiopathic pulmonary fibrosis in smokers $(15,17-21)$. Given its high global frequency of 0.46 in the population according to the Short Genetic Variations 
database (dbSNP) by the National Center for Biotechnology Information (22), we hypothesized that this single-nucleotide polymorphism most likely becomes detrimental only under certain disease-predisposing conditions such as transient surfactant deficiency shortly after birth and in interaction with other potentially disease-predisposing genetic variants like those encoding surfactant protein A. Under these conditions, the alleledependent glycosylation status at Asn129 could have an effect on proSP-B stability, processing, secretion, and/or folding and would thus result in altered mature SP-B levels among individuals carrying different genotypes $(15,17)$. In addition to Ile131Thr (rs1130866), other potentially disease-associating SFTPB polymorphisms that have been assumed to have an effect on transcriptional activity or mRNA splicing include two variations in the promoter region ( $\mathrm{rs} 2077079 \mathrm{C} / \mathrm{A}$ at -18 and rs3024791 G/A at -384) and a length variation in intron $4(23-27)$. These polymorphisms could contribute to individual differences in mRNA and protein levels in vivo. The concentration of mature SP-B in the epithelial lining may depend upon the SFTPB genotype, which could have a causal influence on lung function and pulmonary morbidity. Observations in conditional transgenic adult animals indicate that reduced SP-B protein expression and decreased SP-B concentration in bronchoalveolar lavage fluid are associated with risk for respiratory failure, suggesting that there is a critical threshold of $50 \%$ for alveolar SP-B content that is necessary for normal pulmonary function and gas exchange (28).

In this study, we explored the relative levels of SP-B in amniotic fluid collected from pregnancies at the time of delivery for evaluating whether protein levels correlate with any of the SFTPB polymorphisms that are considered functional. In addition, we determined whether the glycosylation status at Asn129 affects the rate of SP-B secretion, reflecting potential differences in SP-B processing or secretion kinetics between the allelic variants. Finally, we analyzed the allelic expression of the SFTPB mRNA in Ile131Thr heterozygous adult lung tissue samples to quantitate the relative expression of the two variants. Our results are consistent with the initial hypothesis that Ile131Thr allele-specific glycosylation of the proSP-B peptide reduces the production or delays the secretion of mature SP-B through a posttranscriptional mechanism.

\section{RESULTS}

\section{Correlation Between Amniotic Fluid SP-B Levels and SFTPB} Polymorphism

We used western analysis for SP-B quantitation, and the validity of the assay was explored as described in the Supplementary Data online. To determine whether a certain SFTPB polymorphism correlates with protein levels in vivo, the SP-B levels in amniotic fluid were plotted against the SFTPB genotypes.

Amniotic fluid SP-B levels differed significantly between the Ile131Thr (rs1130866) genotypes (ANOVA $P=0.013$; KruskalWallis $P=0.016$ ), whereas there were no correlations between protein levels and genotypes with the other polymorphisms (Table 1). The Thr/Thr genotypes showed lower SP-B levels as compared with the other Ile131Thr genotypes $(P<0.05)$. The relative protein level seemed slightly higher in the heterozygous Ile131Thr group than in the homozygous Ile/Ile group, but the difference was not statistically significant $(P>0.1)$, suggesting that the relative SP-B levels were similar in the Ile/Ile and Ile/Thr genotypes. Infants with the $\mathrm{Thr} / \mathrm{Thr}$ genotype tended to have higher incidence (12.5\%) of respiratory distress (transient tachypnea of the newborn, RDS, or pneumothorax) as compared with infants with Ile/Thr (9.6\%) or Ile/Ile (8.0\%) genotypes, but the number of such infants was small, and this difference was not statistically significant $(P=0.712)$. Infants with respiratory distress had lower SP-B levels than controls $(P<0.001)$ with mean/ median (SE) SP-B levels of 38.3/26.0 (7.6) and 63.8/56.0 (2.6), respectively (see Supplementary Figure S1 online).

Linear regression analyses, taking into account the effects of multiple variables simultaneously, showed that the homozygosity

Table 1. The relative SP-B protein levels in amniotic fluid among the SFTPB genotype groups, showing statistically significant correlation between the genotypes and SP-B levels for rs1130866 but not for the other SFTPB polymorphisms

\begin{tabular}{|c|c|c|c|c|c|c|c|}
\hline Polymorphism & Genotype & $n$ & Mean & ANOVA $P$ value & Median & SE & Kruskal-Wallis $P$ value \\
\hline \multirow[t]{2}{*}{ rs3024791 (G/A at -384) } & $\mathrm{AA}$ & 1 & 65.00 & 0.531 & 65 & 0 & 0.594 \\
\hline & AG & 40 & 55.20 & & 51.5 & 5.49 & \\
\hline \multirow[t]{2}{*}{ rs2077079 (C/A at -18) } & AA & 102 & 60.33 & 0.228 & 53.5 & 3.97 & 0.295 \\
\hline & $A C$ & 117 & 59.66 & & 52 & 3.60 & \\
\hline \multirow[t]{3}{*}{ rs1130866 (Ile131Thr) } & Ile/lle (TT) & 75 & 61.44 & $0.013^{*}$ & 53 & 4.33 & $0.016^{*}$ \\
\hline & Ile/Thr (CT) & 125 & 66.44 & & 61 & 3.67 & \\
\hline & Thr/Thr (CC) & 48 & 49.13 & & 41 & 5.40 & \\
\hline \multirow[t]{2}{*}{ Intron 4 length variation } & Invar/Invar & 192 & 61.66 & 0.952 & 53 & 2.94 & 0.926 \\
\hline & Invar/Del ${ }^{\mathrm{b}}$ & 51 & 60.18 & & 59 & 4.85 & \\
\hline
\end{tabular}

ANOVA, analysis of variance; SP-B, surfactant protein B.

alnvar, invariant allele (wild type). ${ }^{\text {b }}$ el, deletion variant allele. * Significant at $P<0.05$. 
of the Ile131Thr Thr allele (i.e., a recessive inheritance model where Thr/Thr group was compared against the combined Ile/Thr and Ile/Ile group), along with two other covariates gestational age $(P$ $=0.001)$ and respiratory distress $(P=0.001)$, was a significant covariate for SP-B levels in amniotic fluid $(P=0.012)$. The other SFTPB genotypes and other factors (sex and birth weight) did not explain the SP-B levels, nor did they modify the effect of the $S F T P B$ Thr/Thr genotype in adjusted models (data not shown).

\section{SP-B N-Glycosylation and Secretion Kinetics}

Both Chinese hamster ovary $(\mathrm{CHO})$ cell lines that were generated to express one of the two human SFTPB Ile131Thr allelic variants showed robust proSP-B production and secretion in the culture medium. A prominent band of $\sim 42 \mathrm{kDa}$ corresponding to proSP-B was readily detectable in cell lysates and media (see Supplementary Figure S2 online). Both recombinant proteins, proSP-B/Ile and proSP-B/Thr, were sensitive to peptide- $\mathrm{N}$-glycosidase $\mathrm{F}$ due to $\mathrm{COOH}$-terminal glycosylation at Asn311. However, the size shift was larger for proSP-B/Thr, consistent with the presence of an additional oligosaccharide in its $\mathrm{NH}_{2}$ terminus at Asn129 that is absent in proSP-B/Ile (see Supplementary Figure S2 online). These results provide evidence for allele-specific differences in the N-glycosylation of the proSP-B in CHO cells. This allele-specific glycosylation pattern is also seen in human lungs (15).

In pulse-chase labeling experiments with six paired replicates, proSP-B accumulation was detected in the medium in a time-dependent manner lagging 30-60 min behind detection in the cell lysate (Figure 1a). The midpoint times of secretion, illustrated by the V50 values, were $51.9 \pm 1.3$ and $58.2 \pm 1.2 \mathrm{~min}$ for proSP-B/Ile and proSP-B/Thr, respectively (Figure 1b). The results of the pulse-chase labeling experiments demonstrate that in $\mathrm{CHO}$ cells stably expressing proSP-B, the accumulation of $\mathrm{NH}_{2}$-terminal $\mathrm{N}$-glycosylated proSP-B/Thr in the culture medium was delayed by $\sim 12 \%$ as compared with nonglycosylated proSP-B/Ile $(P=0.0012)$.

\section{Allelic Expression Imbalance Assay}

To determine whether the observed differences in the previous experiments arose transcriptionally or posttranscriptionally, we performed an allelic expression imbalance (AEI) assay using RNA from Ile131Thr heterozygous human adult lung tissue samples $(n=8)$. The expression ratio of the Ile and Thr variant mRNAs (multiple replicates), normalized by the reference sample ratio (plasmid DNA, 50:50), was 0.948 \pm 0.111 (mean \pm SD; range 0.792-1.165), which did not differ from the expected value of $1.0(P=0.229)$ (see Supplementary Figure S3 online). This indicated equal mRNA expression and lack of AEI between the two variants.

\section{DISCUSSION}

When studying complex traits, it is challenging to identify the causal risk variants and demonstrate the function of the implicated polymorphisms. Consequently, converting the results of both candidate gene and genome-wide association studies into precise molecular mechanisms and clinical applications is
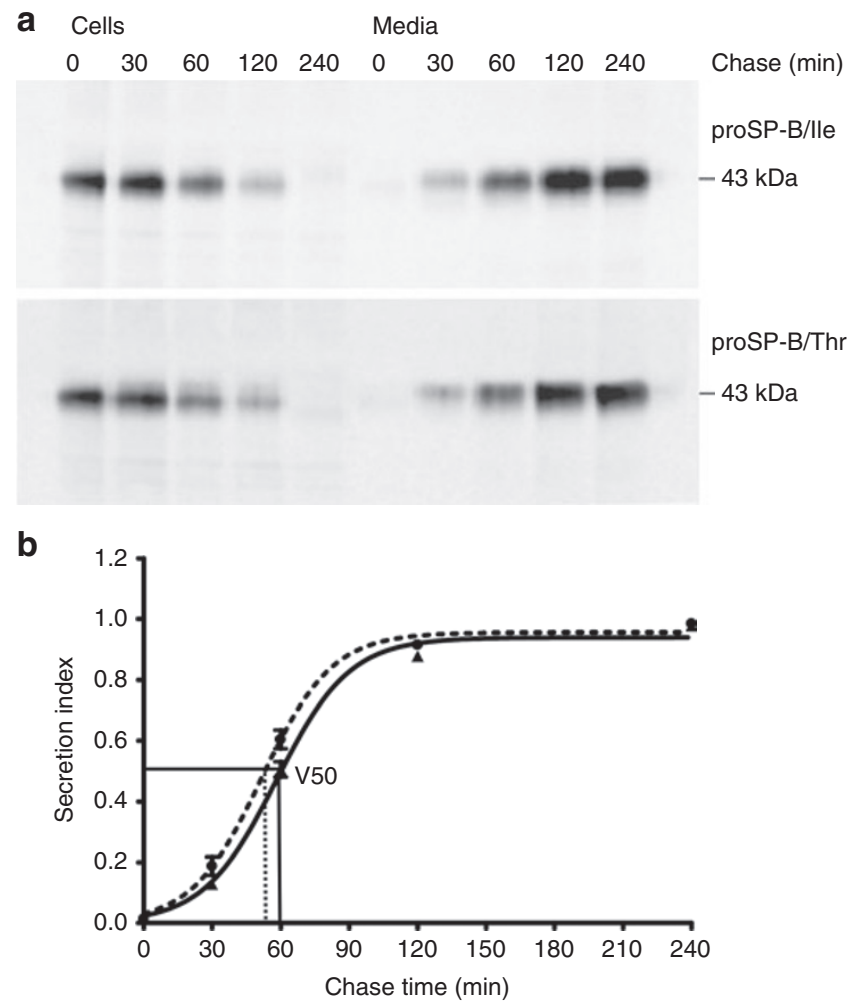

Figure 1. Allele-specific human proSP-B secretion kinetics in transfected Chinese hamster ovary $(\mathrm{CHO})$ cells. Cells were pulse labeled for $30 \mathrm{~min}$ and chased for $0,30,60,120$, and $240 \mathrm{~min}$. Both cells and media were harvested and proSP-B was recovered by immunoprecipitation. Representative phosphorimages are shown for both proSP-B lle and Thr variants. (a) No proSP-B was seen in the medium until at the 30 -min chase time point. Over time, proSP-B accumulated in the medium and the newly synthesized proSP-B was completely secreted from the cells within $240 \mathrm{~min}$. (b) For curve fitting, the chase time was plotted against the secretion index. The secretion index, which describes the proportion of labeled proSP-B in the medium out of total labeled proSP-B, was calculated by dividing the trace quantity value of proSP-B in the medium by the sum of the corresponding values in the medium and cells. The data represent the mean \pm SE from six independent experiments; black circles indicate proSP-B/lle and black triangles indicate proSP-B/Thr. The value at 240-min time point was fixed to 1. The data were fitted to a Boltzmann sigmoidal model; dashed line and continuous line indicates proSP-B/lle and proSP-B/Thr, respectively. V50 values, which illustrate the midpoint of secretion, were $51.9 \pm 1.3$ and $58.2 \pm 1.2 \mathrm{~min}$ for proSP-B/lle and proSP$\mathrm{B} / \mathrm{Th}$, respectively. Glycosylated proSP-B/Thr was secreted from $\mathrm{CHO}$ cells at a significantly lower rate than that of proSP-B/lle $(P=0.0012)$. SP-B, surfactant protein B.

a slow process. The aim of this study was to perform molecular phenotyping for providing evidence for a common variant of SFTPB in disease predisposition. RDS presents a transient developmental deficiency in alveolar surfactant components shortly after premature birth (29). We and others have previously reported that there are associations of SFTPB polymorphisms with RDS and other lung diseases (15-21,25-27). We have also hypothesized a causal role for the Ile131Thr substitution, which selectively alters Asn129 N-glycosylation of the SP-B proprotein $(15,17)$. In this study, this glycosylation showed delayed proSP-B secretion in vitro and an association with decreased extracellular SP-B concentration in vivo. 
The analyses between amniotic fluid SP-B concentration and SFTPB polymorphisms revealed an associating polymorphism: Ile131Thr (rs1130866). In individuals with the homozygous $\mathrm{Thr} / \mathrm{Thr}$ fetal genotype, we detected relatively lower levels of SP-B protein in amniotic fluid at the time of birth. SP-B production is well known to increase from preterm toward term gestation resulting in a rise in the amniotic fluid SP-B concentrations $(30,31)$. Given this, our finding is of specific interest considering that the focus of these analyses was to investigate the term pregnancies without specific clinical complications and which were expected to show high SP-B concentrations. It remains to be explored whether extending these analyses to preterm deliveries with lower amniotic fluid SP-B concentrations would yield a stronger correlation between the genotype and the protein level.

To provide a more mechanistic explanation for these findings, we investigated the effect of the N-glycosylation status at Asn129 on SP-B secretion kinetics using commercially available $\mathrm{CHO}$ cells for generating stably transfected allelespecific proSP-B variant cell lines. Glycosylated proSP-B/Thr was secreted from the $\mathrm{CHO}$ cells at a significantly lower rate than that of proSP-B/Ile. This could be associated with the decreased levels of SP-B that were observed in the amniotic fluid samples with fetal Thr/Thr genotypes. The fact that the in vitro observations were obtained in a cell model lacking a regulated secretory pathway and cell type-specific processing steps implies that the underlying diverging molecular events occur during very early steps. The $\mathrm{NH}_{2}$-terminal peptide containing the Asn 129 site is removed from proSP-B very soon posttranslationally already in the ER (11). This step is not cell specific, as it has also been shown to occur in constantly secreting cells normally not expressing SP-B (16). As a whole, these data are consistent with the view that in type 2 cells, the relevant determinants directing the differential fate of the mature SP-B molecules derived from the variant proSP-B peptides should act very soon posttranslationally. Therefore, the $\mathrm{CHO}$ cell model could provide supporting mechanistic evidence for the functional role of the glycosylation status of Asn129 present in the Thr variant. However, proSP-B is not secreted from type 2 cells and therefore it is not straightforward to generalize the data from $\mathrm{CHO}$ cells to SP-B secretion in vivo. Nevertheless, these in vitro results supplemented with in vivo data using the amniotic fluid samples point to the same direction, i.e. delay in the intracellular events in the processing, sorting or secretion of the glycosylated allelic variant. Together these findings suggest a causal role for the single-nucleotide polymorphism rs1130866 in vivo. However, it remains in question whether these results can be repeated on alveolar type 2 cells that are capable of handling all the steps involved in the processing and packaging of SP-B in vivo.

To exclude the possibility that the Thr variant-associated decreased extracellular SP-B concentration in amniotic fluid and the reduced secretion rate in transfected cells was due to altered transcriptional activity of the variant, we performed an AEI assay in which the allelic ratios of the mRNA variants transcribed in adult human lungs were analyzed. The ratios for the Ile and Thr allele products did not deviate significantly from 1. This suggests that the intrinsic transcription levels of the Ile131Thr variants are equal and the differences in protein levels that we observed are not due to the allele-specific preferences in mRNA expression.

$\mathrm{N}$-glycosylation is known to have a definitive role in governing protein secretion, and it is thought to enhance the folding, trafficking, and stability of glycoproteins by targeting the use of ER folding cycles via chaperones (32). Defective glycosylation caused by mutations typically leads to mislocalization, ER retention, and ER-associated protein degradation, whereas glycosylation sites have also been shown to facilitate improved protein trafficking (33-36). Glycosylation of proSP-B represents a different paradigm. Instead of an infrequent mutation resulting in disease, Ile131Thr results from a single-nucleotide polymorphism in which the potentially unfavorable glycosylated Thr variant allele occurs at a high frequency in the population. Both of these variants allow for processing of fully functional mature protein. Therefore, although the glycosylated Thr variant of SP-B may have different properties compared with the wild-type Ile variant, it is apparently, e.g., not too aberrant to be recognized as a misfolded protein in the ER and destined for proteasomal degradation. It is plausible that the proSP-B Thr variant undergoes slight ER retention, possibly through a prolonged interaction with an ER chaperone protein that assists in folding. This would be adequate for explaining the subtle delay in secretion observed in our study. Of note, when two N-glycosylation sites of the human $\delta$-opioid receptor were mutated, enhanced export of the non-N-glycosylated receptors from the ER was observed (37). This effect on kinetics by glycosylation is similar to what we observed in our study.

As for possible limitations in this study, western blot quantitation with infrared fluorescence is suboptimal in its accuracy for diagnostics, but yet it can be considered adequate for correlation studies not aiming at precise absolute quantitation. Western blot-based quantitation enabled us to distinguish between all proSP-B intermediates and mature SP-B and helped us to overcome the background due to nonspecific binding, typical of enzyme-linked immunosorbent assaybased SP-B quantitation method. Protein quantitation by western immunoblotting with an antibody directly labeled with a near-infrared dye has been shown to be sensitive, linear, and reproducibly quantifiable over a wide range of concentrations $(38,39)$. In addition, the total volume of amniotic fluid varies highly between pregnancies causing intrinsic variation in protein concentrations. This is evident as high variation in SP-B levels in the unaffected subjects in our study. Despite this basal background variation, statistically significant correlations were observed between the protein levels and the genotype. Taking into account the limitations of the quantitation method and variability in amniotic fluid volumes, these analyses are not expected nor meant to provide us with diagnostic value.

In summary, we observed in vivo lower concentrations of mature SP-B secreted from fetal lungs into amniotic fluid in rs1130866 $\mathrm{Thr} / \mathrm{Thr}$ homozygotes compared with the other genotypes. These findings were further supported by in vitro 
experiments showing delayed secretion of the glycosylated proSP-B/Thr variant compared with the nonglycosylated proSP-B/Ile variant in transfected cells. No allelic imbalance was evident in heterozygous Ile/Thr lung tissue samples, suggesting a posttranscriptional mechanism. Together these data suggest that the presence of the $\mathrm{NH}_{2}$-terminal $\mathrm{N}$-glycosylation site due to the amino acid substitution at proSP-B position 131 may be a genetic determinant of delayed passage of the protein through the secretory pathway and decreased extracellular SP-B concentrations. This effect is not substantial enough to decrease the SP-B protein contents below the critical level, which is a decrease by $>50 \%$ of the normal alveolar SP-B content (28), for normal pulmonary function under stable conditions. However, even a smaller decrease could serve as a trigger for transient respiratory distress during the critical first few minutes of life after premature birth, when maintenance of adequate alveolar volumes for normal efficient breathing and gas exchange needs to start immediately with no delay in the optimal secretion of surfactant constituents. As a conclusion, our study provides mechanistic evidence to support a causal role of the SFTPB Ile131Thr polymorphism, which has previously been shown in several studies to associate with genetic susceptibility to pulmonary outcomes, such as RDS.

\section{METHODS}

Detailed description of the materials and methods are available in the Supplementary Data online.

\section{Sample Collection and Study Population}

Sample pairs of amniotic fluid and umbilical cord blood $(n=251)$ were obtained from elective cesarean section deliveries of term singleton pregnancies with gestation ranging from 37 to $40 \mathrm{wk}$. The characteristics of the study population are shown in Table 2. Detailed diagnostic criteria for respiratory symptoms are presented in the Supplementary Data online. Adult human lung tissue was obtained from patients undergoing lung surgery. Informed consent was obtained from all the subjects. The study was approved by the Ethics Committee of the Oulu University Hospital.

\section{Amniotic Fluid Sample Preparation and Western Analysis}

Proteins from amniotic fluid samples were separated with sodium dodecyl sulfate-polyacrylamide gel electrophoresis. A pooled amniotic fluid sample known to contain high levels of SP-B was used as a standardized reference in each western blot. The western quantitation assay was explored for reproducibility, linearity, level of detection, and sensitivity as described in the Supplementary Data online.

\section{Genotyping of SFTPB Gene Polymorphisms}

Genotyping of SFTPB Ile131Thr (rs1130866) polymorphism and $\Delta \mathrm{i} 4$ was performed as described previously (17). For $\mathrm{C} / \mathrm{A}(-18)$

Table 2. The characteristics of the study population used for amniotic fluid SP-B quantitation

\begin{tabular}{lrcccc}
\hline & & & \multicolumn{3}{c}{ Gestational age $(w k+d)$} \\
\cline { 4 - 6 } & $n$ & Male/female & Mean & Median & Range \\
\hline All infants & 251 & $137 / 114$ & $38+6$ & $39+0$ & $37+0$ to $40+6$ \\
TTN & 19 & $12 / 7$ & $38+4$ & $38+4$ & $37+1$ to $40+0$ \\
RDS & 1 & $0 / 1$ & $37+5$ & $37+5$ & $37+5$ \\
Pneumothorax & 3 & $2 / 1$ & $38+6$ & $38+6$ & $38+3$ to $39+3$ \\
\hline
\end{tabular}

RDS, respiratory distress syndrome; SP-B, surfactant protein $B ; T T N$, transient tachypnea of the newborn. (rs2077079) and A/G(-384) (rs3024791), primer pairs and restriction enzymes were designed for polymerase chain reaction and restriction fragment length polymorphism analyses as described in the Supplementary Data online.

Reverse Transcription PCR, Construction of Plasmids, and Generation of Stably Transfected ProSP-B-Expressing CHO Cells RNA from human lung tissue heterozygous for SFTPB Ile131Thr was reverse transcribed to cDNA and amplified by polymerase chain reaction. The $1,146 \mathrm{bp}$ reverse transcriptase-polymerase chain reaction products were subcloned, and the resulting expression constructs containing the Ile or Thr variant cDNA were transfected into Flp-In$\mathrm{CHO}$ cells (Invitrogen, Carlsbad, CA). Cells were cultured and the SP-B-expressing clones were selected. Justification for the use of $\mathrm{CHO}$ cells, instead of using cells such as alveolar type 2 cells, is described in detail in the Supplementary Data online.

\section{ProSP-B Detection in CHO Cells and Carbohydrate Removal With} Peptide-N-glycosidase $\mathrm{F}$

Cells and culture media were assayed for SP-B/Ile and SP-B/Thr expression and secretion by western blot analysis. Peptide-Nglycosidase $\mathrm{F}$ digestion was performed to confirm the presence of asparagine-linked oligosaccharides in the expressed proSP-B variants.

Pulse-Chase Labeling, Immunoprecipitation, and Immuno-blotting $\mathrm{CHO}$ cells stably expressing either proSP-B/Ile or proSP-B/Thr were transferred from Ham's F-12 to Dulbecco's modified Eagle's medium (Sigma-Aldrich, St Louis, MO). Nontransfected CHO cells were used as control for absence of SP-B. After a 30-min pulse, both cells and media were harvested at intervals of $0,30,60,120$, and 240 min during the 240-min chase. Radiolabeled CHO cell pellets were lysed, and both lysates and media were used for immunoprecipitation to analyze the kinetics of secretion.

\section{AEl Assay}

cDNA from adult human lung tissue samples $(n=8)$ heterozygous for the SFTPB Ile131Thr polymorphism were tested for AEI using SNaPshot assay (Applied Biosystems, Foster City, CA).

\section{Statistical Analyses}

Statistical analyses were performed using PASW Statistics 18 Data Editor (LEAD Technologies, Charlotte, NC). Parametric (ANOVA) and nonparametric (Kruskal-Wallis) tests were used for analyzing the correlation between SP-B protein levels and genotypes. Linear regression was used for multivariate analysis and one-sample $t$-test for AEI analysis. A $P$ value of $<0.05$ was considered significant.

\section{SUPPLEMENTARY MATERIAL}

Supplementary material is linked to the online version of the paper at http://www.nature.com/pr

\section{ACKNOWLEDGMENTS}

We thank Maarit Haarala, Mirkka Ovaska, Panu Taskinen, and Riitta Vikeväinen for their technical assistance and sample collection and Maritta Pietilä for her methodological advice.

\section{STATEMENT OF FINANCIAL SUPPORT}

This work was supported by the Academy of Finland (R.H. and M.H.), Foundation of Pediatric Research in Finland (R.H.), Päivikki and Sakari Sohlberg Foundation (R.H.), Sigrid Juselius Foundation (M.H.), and HL059959 (S.H.G.) from the National Institutes of Health (Bethesda, MD).

\section{REFERENCES}

1. Griese M. Pulmonary surfactant in health and human lung diseases: state of the art. Eur Respir J 1999;13:1455-76.

2. Rooney SA, Young SL, Mendelson CR. Molecular and cellular processing of lung surfactant. FASEB J 1994;8:957-67.

3. Chroneos ZC, Sever-Chroneos Z, Shepherd VL. Pulmonary surfactant: an immunological perspective. Cell Physiol Biochem 2010;25:13-26. 
4. Nogee LM, Garnier G, Dietz HC, et al. A mutation in the surfactant protein $\mathrm{B}$ gene responsible for fatal neonatal respiratory disease in multiple kindreds. J Clin Invest 1994;93:1860-3.

5. Clark JC, Wert SE, Bachurski CJ, et al. Targeted disruption of the surfactant protein B gene disrupts surfactant homeostasis, causing respiratory failure in newborn mice. Proc Natl Acad Sci USA 1995;92:7794-8.

6. Guttentag S. Posttranslational regulation of surfactant protein B expression. Semin Perinatol 2008;32:367-70.

7. Weaver TE, Conkright JJ. Function of surfactant proteins B and C. Annu Rev Physiol 2001;63:555-78.

8. van Iwaarden JF, Claassen E, Jeurissen SH, Haagsman HP, Kraal G. Alveolar macrophages, surfactant lipids, and surfactant protein B regulate the induction of immune responses via the airways. Am J Respir Cell Mol Biol 2001;24:452-8.

9. Epaud R, Ikegami M, Whitsett JA, Jobe AH, Weaver TE, Akinbi HT. Surfactant protein B inhibits endotoxin-induced lung inflammation. Am J Respir Cell Mol Biol 2003;28:373-8.

10. Beers MF, Shuman H, Liley HG, et al. Surfactant protein B in human fetal lung: developmental and glucocorticoid regulation. Pediatr Res 1995;38:668-75.

11. Guttentag SH, Beers MF, Bieler BM, Ballard PL. Surfactant protein B processing in human fetal lung. Am J Physiol 1998;275(3 Pt 1):L559-66.

12. Guttentag S, Robinson L, Zhang P, Brasch F, Bühling F, Beers M. Cysteine protease activity is required for surfactant protein $\mathrm{B}$ processing and lamellar body genesis. Am J Respir Cell Mol Biol 2003;28:69-79.

13. Korimilli A, Gonzales LW, Guttentag SH. Intracellular localization of processing events in human surfactant protein B biosynthesis. J Biol Chem 2000;275:8672-9.

14. Breslin JS, Weaver TE. Binding, uptake, and localization of surfactant protein B in isolated rat alveolar type II cells. Am J Physiol 1992;262(6 Pt 1):L699-707.

15. Marttila R, Haataja R, Guttentag S, Hallman M. Surfactant protein A and $\mathrm{B}$ genetic variants in respiratory distress syndrome in singletons and twins. Am J Respir Crit Care Med 2003;168:1216-22.

16. Wang G, Christensen ND, Wigdahl B, Guttentag SH, Floros J. Differences in N-linked glycosylation between human surfactant protein-B variants of the $\mathrm{C}$ or $\mathrm{T}$ allele at the single-nucleotide polymorphism at position 1580 : implications for disease. Biochem J 2003;369(Pt 1):179-84.

17. Haataja R, Rämet M, Marttila R, Hallman M. Surfactant proteins A and $\mathrm{B}$ as interactive genetic determinants of neonatal respiratory distress syndrome. Hum Mol Genet 2000;9:2751-60.

18. Lin Z, Pearson C, Chinchilli V, et al. Polymorphisms of human SP-A, SP-B, and SP-D genes: association of SP-B Thr131Ile with ARDS. Clin Genet 2000;58:181-91.

19. Selman M, Lin HM, Montaño M, et al. Surfactant protein A and B genetic variants predispose to idiopathic pulmonary fibrosis. Hum Genet 2003;113:542-50.

20. Quasney MW, Waterer GW, Dahmer MK, et al. Association between surfactant protein $\mathrm{B}+1580$ polymorphism and the risk of respiratory failure in adults with community-acquired pneumonia. Crit Care Med 2004;32:1115-9.

21. Floros J, Fan R, Diangelo S, Guo X, Wert J, Luo J. Surfactant protein (SP) $\mathrm{B}$ associations and interactions with SP-A in white and black subjects with respiratory distress syndrome. Pediatr Int 2001;43:567-76.
22. Sherry ST, Ward M, Sirotkin K. dbSNP-database for single nucleotide polymorphisms and other classes of minor genetic variation. Genome Res 1999;9:677-9.

23. Steagall WK, Lin JP, Moss J. The C/A(-18) polymorphism in the surfactant protein $\mathrm{B}$ gene influences transcription and protein levels of surfactant protein B. Am J Physiol Lung Cell Mol Physiol 2007;292:L448-53.

24. Thomas KH, Meyn P, Suttorp N. Single nucleotide polymorphism in 5 -flanking region reduces transcription of surfactant protein $\mathrm{B}$ gene in H441 cells. Am J Physiol Lung Cell Mol Physiol 2006;291:L386-90.

25. Floros J, Veletza SV, Kotikalapudi P, et al. Dinucleotide repeats in the human surfactant protein-B gene and respiratory-distress syndrome. Biochem J 1995;305 (Pt 2):583-90.

26. Makri V, Hospes B, Stoll-Becker S, Borkhardt A, Gortner L. Polymorphisms of surfactant protein $B$ encoding gene: modifiers of the course of neonatal respiratory distress syndrome? Eur J Pediatr 2002;161:604-8.

27. Rova M, Haataja R, Marttila R, Ollikainen V, Tammela O, Hallman M. Data mining and multiparameter analysis of lung surfactant protein genes in bronchopulmonary dysplasia. Hum Mol Genet 2004;13:1095-104.

28. Melton KR, Nesslein LL, Ikegami M, et al. SP-B deficiency causes respiratory failure in adult mice. Am J Physiol Lung Cell Mol Physiol 2003;285:L543-9.

29. Hallman M, Merritt TA, Akino T, Bry K. Surfactant protein A, phosphatidylcholine, and surfactant inhibitors in epithelial lining fluid. Correlation with surface activity, severity of respiratory distress syndrome, and outcome in small premature infants. Am Rev Respir Dis 1991;144:1376-84.

30. Pryhuber GS, Hull WM, Fink I, McMahan MJ, Whitsett JA. Ontogeny of surfactant proteins A and B in human amniotic fluid as indices of fetal lung maturity. Pediatr Res 1991;30:597-605.

31. Dilger I, Schwedler G, Dudenhausen JW. Determination of the pulmonary surfactant-associated protein SP-B in amniotic fluid with a competition ELISA. Gynecol Obstet Invest 1994;38:24-7.

32. Aebi M, Bernasconi R, Clerc S, Molinari M. N-glycan structures: recognition and processing in the ER. Trends Biochem Sci 2010;35:74-82.

33. Isosomppi J, Västinsalo H, Geller SF, Heon E, Flannery JG, Sankila EM. Disease-causing mutations in the CLRN1 gene alter normal CLRN1 protein trafficking to the plasma membrane. Mol Vis 2009;15:1806-18.

34. Antenos M, Stemler M, Boime I, Woodruff TK. N-linked oligosaccharides direct the differential assembly and secretion of inhibin alpha- and betaAsubunit dimers. Mol Endocrinol 2007;21:1670-84.

35. Bas T, Gao GY, Lvov A, Chandrasekhar KD, Gilmore R, Kobertz WR. Posttranslational N-glycosylation of type I transmembrane KCNE1 peptides: implications for membrane protein biogenesis and disease. J Biol Chem 2011;286:28150-9.

36. Vogt G, Vogt B, Chuzhanova N, Julenius K, Cooper DN, Casanova JL. Gain-of-glycosylation mutations. Curr Opin Genet Dev 2007;17:245-51.

37. Markkanen PM, Petäjä-Repo UE. N-glycan-mediated quality control in the endoplasmic reticulum is required for the expression of correctly folded delta-opioid receptors at the cell surface. J Biol Chem 2008;283:29086-98.

38. Gerk PM. Quantitative immunofluorescent blotting of the multidrug resistance-associated protein 2 (MRP2). J Pharmacol Toxicol Methods 2011;63:279-82.

39. Schutz-Geschwender A, Zhang Y, Holt T, McDermitt D, Olive D. Quantitative, two-color western blot detection with infrared fluorescence. LI-COR Biosciences, 2004. (http://www.licor.com/bio/PDF/IRquant.pdf.) 